\begin{tabular}{|c|c|c|}
\hline Beitr. Ent. & Keltern & ISSN 0005-805X \\
\hline $\mathbf{5 8}(2008) 1$ & S. $135-144$ & 15.07 .2008 \\
\hline
\end{tabular}

\title{
A revision of the Habrocerinae of the world. IV. A new species of Habrocerus from China and additional records
}

\section{(Coleoptera: Staphylinidae)}

With 11 figures and 1 map

\author{
VOLKer AsSing
}

\section{Summary}

Habrocerus splendens sp. n. (China: Yunnan) of the H. capillaricornis group is described and illustrated. Additional records of Habrocerinae are reported, among them first records of $H$. pisidicus Korge from France and Croatia, of H. cyprensis Assing \& Wunderle from Turkey, and a female-based first record of H. ibericus Assing \& Wunderle from Morocco.

Key words

Coleoptera, Staphylinidae, Habrocerinae, Habrocerus, Nomimocerus, world, China, taxonomy, new species, new records.

New species

Habrocerus splendens sp. n.

\section{Zusammenfassung}

Habrocerussplendenssp. n. (China:Yunnan) aus der H. capillaricornis-Gruppe wird beschrieben und abgebildet. Weitere Nachweise von Arten der Habrocerinae werden gemeldet, darunter mehrere Erstnachweise von H. pisidicus Korge aus Frankreich und Kroatien, von H. cyprensis Assing \& Wunderle aus der Türkei und von H. ibericus Assing \& WunderLe aus Marokko; Letzterer basiert allerdings auf Weibchen.

\section{Introduction}

The Habrocerinae has been comprehensively revised in three earlier contributions (Assing \& Wunderle 1995, 1996; Assing 1998). The small subfamily previously included 21 species worldwide, 15 in the genus Habrocerus Erichson and 6 in Nomimocerus Coiffait \& Saiz. Herman (2001) and Newton (2006) indicate 22 Habrocerus species, but this figure includes "H." magnus from North America, which was explicitly excluded from the genus and the subfamily by Assing \& Wunderle (1995); the species may in fact refer to the Tachyporinae.

While Nomimocerus is confined to the Chilean subregion, Habrocerus is more widely distributed originally in the Palaearctic, the Oriental, the Nearctic and the Neotropical regions, with one species, H. capillaricornis (Gravenhorst), of almost cosmopolitan distribution and introduced in the Ethiopian, Neotropical, Nearctic, and Australian regions. Only one species, H. schuelkei Assing \& Wunderle, was previously known from China. 
During a joint recent field trip to China, Michael Schülke, David Wrase (both Berlin), and Andreas Pütz (Eisenhüttenstadt) discovered an undescribed Habrocerus in western Yunnan, the first new species of Habrocerinae in almost a decade. I use the opportunity to report additional records that have been compiled since the last contribution.

\section{Material and methods}

The material referred to below is deposited in the following collections:

$\begin{array}{ll}\text { DEI } & \text { Deutsches Entomologisches Institut, Müncheberg (L. Zerche, L. Behne) } \\ \text { MNHUB } & \text { Museum für Naturkunde der Humboldt-Universität Berlin (J. Frisch) } \\ \text { NHMW } & \text { Naturhistorisches Museum Wien (H. Schillhammer) } \\ \text { SMNS } & \text { Staatliches Museum für Naturkunde, Stuttgart (W. Schawaller) } \\ \text { cAng } & \text { private collection Fernando Angelini, Francavilla Fontana } \\ \text { cAss } & \text { author's private collection } \\ \text { cDro } & \text { private collection Bozo Drovenik, Ljubljana } \\ \text { cGar } & \text { private collection Rafael García Becerra, Santa Cruz de La Palma } \\ \text { cHir } & \text { private collection Gunnar Hirthe, Mühl-Rosin } \\ \text { cMac } & \text { private collection Antonio Machado, La Laguna } \\ \text { cMif } & \text { private collection David Mifsud, Malta } \\ \text { cPüt } & \text { private collection Andreas Pütz, Eisenhüttenstadt } \\ \text { cRos } & \text { private collection Armin Rose, Oldenburg } \\ \text { cSch } & \text { private collection Michael Schülke, Berlin } \\ \text { cZan } & \text { private collection Adriano Zanetti, Verona }\end{array}$

The morphological studies were carried out using a Stemi SV 11 microscope (Zeiss Germany) and a Jenalab compound microscope (Carl Zeiss Jena). For the photographs a digital camera (Nikon Coolpix 995) was used.

The map was generated using the online generic mapping tool (GMT) of the Geomar website at www. aquarius.ifm-geomar.de/omc.

The nomenclature of the male and female terminalia follows Assing \& WUNDerLe (1995).

\section{Additional records and species description}

\section{H. capillaricornis (GRAVENHORST)}

Additional material examined:

Tunisia: 4 exs., ca. $30 \mathrm{~km}$ E Siliana, Bou Saadia, 360.' $\mathrm{N}$, 09³ $38^{\prime} \mathrm{E}, 550 \mathrm{~m}$, N-slope with macchia, litter and grass sifted, 25.XII.2004, leg. Assing (cAss); 1 ex., ca. 2 km S Aïn Draham, 36' $44^{\prime} \mathrm{N}, 08^{\circ} 41^{\prime} \mathrm{E}, 670 \mathrm{~m}$, oak forest, litter sifted, 28.XII.2004, leg. Assing (cAss).

Morocco: 4 exs., Beni Snassen Mts., $10 \mathrm{~km} \mathrm{~S}$ Berklane, Zegzel, $34^{\circ} 50^{\prime} \mathrm{N}, 2^{\circ} 22^{\prime} \mathrm{W}, 400 \mathrm{~m}$, arable land, 30.XII.2001, leg. Bayer (cSch, cAss); 1 ex., Haut Atlas, 40 km SSE Marrakech, Arhbalou, 31 ${ }^{\circ} 19^{\prime} \mathrm{N}, 07^{\circ} 45^{\prime} \mathrm{W}$, 1030 m, edge of stream, 28.XII.2002, leg. Assing (cAss).

Spain: mainland: 1 ex., País Vasco, $60 \mathrm{~km}$ SE Bilbao, Sierra de Urquilla, SE Arantzazu, 42 $58^{\prime} \mathrm{N}, 02^{\circ} 23^{\prime} \mathrm{W}$, 860 m, beech forest, 10.VII.2003, leg. Assing (cAss); 1 ex., Vera de Bidasoa, Col d'Ibardin, 200 m, 19.V.1990, leg. Hartmann (DEI); 1 ex., Galicia, Sierra de Ancares, ENE Degrada, 4250'N, 65' W, 970 m, mixed deciduous forest with very old Castanea sativa, 14.VII.2004, leg. Assing (cAss); 2 exs., Galicia, Sierra do 
Courel, Rio de Rofión, 23.V.2000, leg. Valcárcel (cSch); 1 ex., Girona, 7 km S Vidreres, 41²43'N, 251'E, 150 m, oak wood with Ilex, 10.X.1997, leg. Zerche; 1 ex., Andalucía, Cerro de la Novia, barranco at km 6 of A-375, 36 $37^{\prime} \mathrm{N}, 5^{\circ} 30^{\prime} \mathrm{W}$, ca. $700 \mathrm{~m}$, collected together with H. ibericus, 19.II.2000, leg. Lompe (cAss). Canary Islands: Gran Canaria: 1 ex., Gran Canaria, S El Cortijo, S Fontanales, 28 $03^{\prime} \mathrm{N}, 15^{\circ} 37^{\prime} \mathrm{W}, 1120 \mathrm{~m}$, 31.I.1998, leg. Zerche (DEI); 1 ex., Bco. Oscuro, 12.VI.1984, leg. Machado (cMac). La Gomera: 103 exs., NE Arure, Arańa, 1000-1100 m, moist litter in laurisilva, 24.XII.1998, leg. Assing (cAss); 6 exs., 4 km NE Arure, S Zarza, 1000 m, laurisilva, 25.XII.1998, leg. Assing (cAss). La Palma: 5 exs., Tagoja, 3.II.1994, leg. García (cGar, cAss); 2 exs., Bco. del Carmen, 5.I.1982, leg. García (cGar); 1 ex., Tagoja, 24.III.1990, leg. Machado (cMac).

Portugal: 1 ex., Algarve, Serra de Monchique, NW Picota, 22.II.1999, leg. Meybohm (cAss).

France: 1 \% , Haute-Corse, Fontane de Padula, $3 \mathrm{~km} \mathrm{~W}$ Vezzani, $42^{\circ} 11^{\prime} \mathrm{N}, 9^{\circ} 13^{\prime} \mathrm{E}, 900 \mathrm{~m}$, moss, 29.VII.1998, leg. Zerche \& Ruch (DEI); 1 \& , Haute-Corse, Castagniccia, San Nicolao, 11.V.2001, leg. Wolf (cSch).

Italy: $1 \sigma^{\star}$, Sardegna, Cagliari, Torredelle, 13.-18.VII.1998, leg. Menzel (DEI); 1 ex., Sicilia, 40 km W Siracusa, NE Buccheri, $37^{\circ} 08^{\prime} \mathrm{N}, 14^{\circ} 53^{\prime} \mathrm{E}, 580 \mathrm{~m}$, oak forest, sifted, 23.XII.2007, leg. Assing (cAss).

Malta: 2 우, 앙 Wied il-Luq, 15.IV. \& 1.V.1990, leg. Mifsud (cMif); 1 우, Buskett Gardens, 30.XII.1994, leg. Zerche (DEI).

Slovakia: 4 exs., E Banovice, E Ostrý Vrach peak, 4844'N, 18²2'E, 520 m, fagetum, 3.VIII.2001, leg. Zerche (DEI).

Hungary: 5 exs., SW Aggtelek, 2 km E Imola, 48²5'N, 20³5'E, 300 m, oakwood, 31.VII.2001, leg. Zerche (DEI, cAss).

Slovenia: 5 exs., Žiče, Sotensko, 23.IV.1998, leg. Drovenik (cDro, cAss); 1 ex., Police, Gor. Radgona, 28.III.1998, leg. Drovenik (cDro); 2 ex., Podorad, Mura, G. Radgona, 17.IV.1996, leg. Drovenik (cDro, cAss); 1 ex., Kamnik, Volčji potok, 6.IV.1996, leg. Drovenik (cDro); 1 ex., Zasip, Sava, 12.IV.1996, leg. Drovenik (cDro); 2 exs., Kocevje, Kocevski Rog, Globocica near Podturn, 500 m, doline, 7.VII.1996, leg. Zerche (DEI).

Croatia: 8 exs., Plitvice, Supljara doline, 510 m, 15.VII.1996, leg. Zerche (DEI).

Romania: 1 ex., Neauesti, 25 km SE Piatra Neamt., 500 m, 18.VI.1996, leg. Prudek (cSch).

Bulgaria: 1 ex., Stara Planina, Aprilzi, N Monastery, 42 $51^{\prime} \mathrm{N}, 2^{\circ} 55^{\prime} \mathrm{E}, 480 \mathrm{~m}$, Carpinus-Ulmus forest, 26.V.2000, leg. Zerche \& Behne (DEI).

Greece: 5 exs., Thessalia, NE Ossa Oros, W Stómio, ca. 20 m, flood plain forest, 5.IV.1998, leg. Assing (cAss); 1 ex., Katara pass, 1500 m, 13.V.1997, leg. Wolf (cSch); 1 ex., Pelopónnisos, 20 km S Tripoli, Vlachokerasia, 27.IV.1999, leg. Wolf (cSch).

Turkey: 3 exs., Antalya, between Antalya and Alanya, Incekum, 16.IV.-2.V.1984, leg. Brachat (cSch).

Azerbaijan: 2 exs., Astara, Istisu W Astara, 100 m, 2.-6.VI.1996, leg. Schawaller (SMNS, cAss).

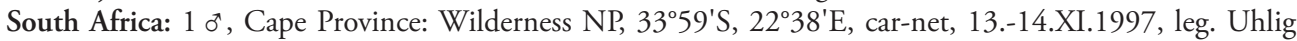
(MNHUB).

The species is most likely of Western Palaearctic origin, but has an almost cosmopolitan distribution today. Outside the Palaearctic region, it has been introduced in the Australian region (New Zealand), as well as in South America (Colombia) and North America (Assing \& Wunderle 1995); recently it was also reported from South Africa for the first time (Newton 2006).

For additional records from La Palma, Canary Islands, where the species is very common in various biotopes, see Assing \& Wunderle (1999). The species is apparently absent from El Hierro, where it was not found in spite of extensive field work in December and January 1999/2000. The records by Franz (1996), according to whom H. capillaricornis is quite common in the island, refer to $H$. canariensis; the corresponding material from the Franz collection was examined (see section on $H$. canariensis). 


\section{Habrocerus pisidicus KORGE}

\section{Additional material examined:}

France: Corse: 7 exs., Corse-du-Sud, Cascade du Voile de la Mariée, 2 km S Bocognano, 4204' N, 0903'E, 720 m, Buxus litter, 8.X.2001, leg. Behne (DEI, cAss).

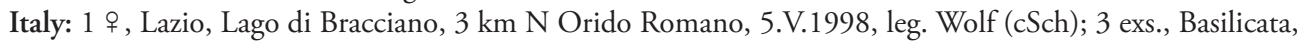
bosco Montepiano (MT), Accetura, 900 m, 29.IX.2002 Angelini (cAng, cAss); 1 o, Sardegna, Cagliari, Iglesia Marganai, $700 \mathrm{~m}$, pitfall trap, 21.IX.-6.X.2004, leg. Chessa (cZan).

Croatia: 2 exs., Makarska Biokovo, Gorna Brela canyon, 21.X.2005, leg. Hlaváč (cAss); 1 ex., Biokovo planina, N Bast, 400-500 m, 20.X.2005, leg. Hlaváč (cAss).

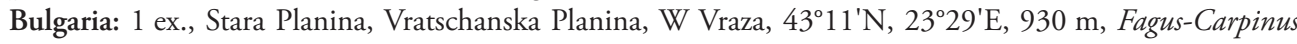
forest, 23.V.2000, leg. Behne (cAss); 3 exs., Stara Planina, Babin Nos, N Rakovischki Manastir, 434' $\mathrm{N}$, $22^{\circ} 25^{\prime} \mathrm{E}, 585 \mathrm{~m}$, Tilia forest, 20.V.2000, leg. Zerche \& Behne (DEI); 1 ex., Stara Planina, Mala Planina NW Kostinbrod, $42^{\circ} 55^{\prime} \mathrm{N}, 23^{\circ} 10^{\prime} \mathrm{E}, 920 \mathrm{~m}$, steppe with shrubs, 16.V.2000, leg. Zerche \& Behne (DEI);

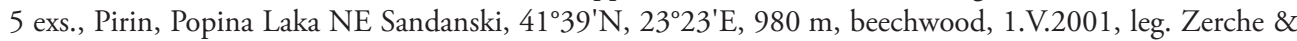

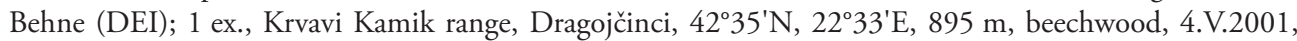
leg. Zerche \& Behne (DEI); 1 ex., Belasiza S Petritsch, $41^{\circ} 22^{\prime} \mathrm{N}, 23^{\circ} 11^{\prime} \mathrm{E}, 720 \mathrm{~m}$, Castanea-Fagus forest, 5.V.2000, leg. Behne (DEI).

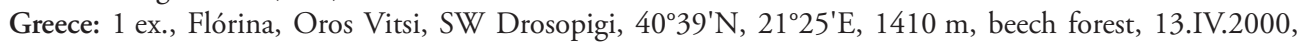
leg. Behne (DEI); 5 exs., N-Pindos, ca. 40 km NNE Konitsa, Oros Gramos range, S Oros Arénes, SE Aetomilitsa, $40^{\circ} 18^{\prime} \mathrm{N}, 20^{\circ} 53^{\prime} \mathrm{E}, 1650 \mathrm{~m}$, beech forest, sifted, 25.V.2005, leg. Assing \& Wunderle (cAss, cWun); 1 ex., Ioannina, ca. $15 \mathrm{~km} \mathrm{SE}$ Konitsa, Oros Timfi, W Vrisochori, 39 $59^{\circ} \mathrm{N}, 20^{\circ} 53^{\prime} \mathrm{E}, 1280 \mathrm{~m}$, beech forest, 26.V.2005, leg. Assing (cAss); 1 ex., Ipiros, 30 km N Igoumenitsa, 11.V.1997, leg. Wolf (cSch); 3 exs., Thessalia, Trikala, Katara pass, $39^{\circ} 48^{\prime} \mathrm{N}, 21^{\circ} 20^{\prime} \mathrm{E}, 680 \mathrm{~m}$, oak forest, 2.IV.2001, leg. Zerche \& Behne (DEI); 1 ex., Drama, Falakró, above Vólakas, $41^{\circ} 17^{\prime} \mathrm{N}, 24^{\circ} 00^{\prime} \mathrm{E}, 1100 \mathrm{~m}$, beech forest, 10.IV.2000, leg. Behne (DEI); 12 exs., Thessalia, Ossa Oros, SE Stómio, 10 0m, 4.IV.1998, leg. Assing (cAss); 1 ex., Ossa Oros, 1000 m, 18.V.1997, leg. Wolf (cSch); 2 exs., Makedhonia, Olympos, NW Stavros, 1400 m, 7.IV.1998, leg. Assing (cAss); 1 ex., Voiotia, Oros Elikonas, road from Kiriaki to Elikonas, 38²3'N, 2249'E, 900 m, Abies forest, 4.IV.2001, leg. Assing (cAss); 1 ex., Makedhonia, Pieria Ori, above Skotina, 900-1000 m, 9.IV.1998, leg. Assing (cAss); 3 exs., Makedhonia, Piéria, above Litochoro, $40^{\circ} 07^{\prime} \mathrm{N}, 22^{\circ} 23^{\prime} \mathrm{E}, 1500 \mathrm{~m}$, 6.IV.2001, leg. Zerche (DEI); 1 ex., Makedhonia, Vermion Oros, above Seli, 1500 m, 11.IV.1998, leg. Assing (cAss); 6 exs., Vermion Oros, Séli ski resort, $40^{\circ} 33^{\prime} \mathrm{N}, 22^{\circ} 00^{\prime} \mathrm{E}, 1490 \mathrm{~m}$, beech forest, 14.IV.2000, leg. Zerche \& Behne (DEI); 1 ex., Vermion Oros, above Kastania, 1300 m, 11.IV.1998, leg. Assing (cAss); 4 exs., Pelopónnisos, Erimanthos Oros, 3.7 km N Plaka, 900m, 5.V.1999, leg. Wolf (cSch); 1 ex., Pelopónnisos, Parnon Oros, W Vamvakou, 800 m, 1.V.1999, leg. Wolf (cSch). Evvoia: 1 ex., Evvoia, Oros Lichas, 3851'N, $22^{\circ} 54^{\prime} \mathrm{E}, 525 \mathrm{~m}$, Pinus forest, 8.IV.2001, leg. Assing (cAss). Rodhós: 1 ㅇ, Arhipolis, valley to Platania, 200 m, 14.IV.1999, leg. Meybohm (cAss); 1 ㅇ, Eleousa Ag. Nikalaos, Fountouldi, 300 m, 9.IV.1999, leg. Meybohm (cAss); 5 exs., Kolimbia Epta Piges, 100 m, 10.IV.1999, leg. Meybohm (cAss). Kárpathos: 10 , Aperi, 200m, 20.IV.1999, leg. Meybohm (cAss). Kríti: 1 옹 Sfinari, 35²4'N, 23³5'E, 240 m, 15.III.2001, leg. Meybohm (cAss). Sámos: 2 exs., Platanakia, 37047'N, 26 50'E, 50-200 m, 21.IV.2003, leg. Meybohm

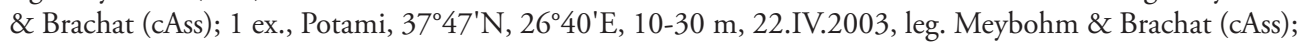

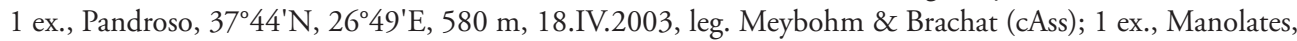
$37^{\circ} 47^{\prime} \mathrm{N}, 26^{\circ} 50^{\prime} \mathrm{E}, 300 \mathrm{~m}, 29 . I V .2003$, leg. Meybohm \& Brachat (cAss); 1 ex., Avlakia, $37^{\circ} 48^{\prime} \mathrm{N}, 26^{\circ} 51^{\prime} \mathrm{E}$, $100 \mathrm{~m}, 28 . \mathrm{IV} .2003$, leg. Meybohm \& Brachat (cAss). Ikaría: $30^{\top} \mathrm{o}^{\top}, \mathrm{Nas}, 37^{\circ} 37^{\prime} \mathrm{N}, 36^{\circ} 03^{\prime} \mathrm{E}, 10-100 \mathrm{~m}$, 26.IV.2003, leg. Meybohm \& Brachat (cAss).

Turkey: Izmir: 3 exs., Boz Dağları, Boz dağ köy, 1200 m, 30.V.-3.VI.2003, leg. Lohaj (cSch); 1 ex., same

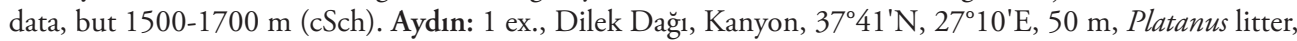
29.III.2006, leg. Meybohm (cAss); 1 ex., Dilek Dağı, S Kanyon, $37^{\circ} 40^{\prime} \mathrm{N}, 27^{\circ} 11^{\prime} \mathrm{E}, 670$ m, 17.IV.2006,

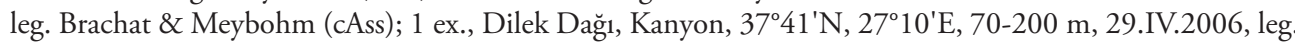

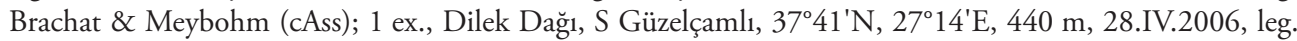
Brachat \& Meybohm (cAss). Denizli: 148 exs. [partly teneral], ca. $50 \mathrm{~km} \mathrm{~N}$ Fethiye, S Çameli, 36 $59^{\prime} \mathrm{N}$, $29^{\circ} 16^{\prime} \mathrm{E}, 1245 \mathrm{~m}$, litter of Quercus ilex, 9.VII.2002, leg. Assing (cAss). Muğla: 11 exs., N Marmaris, 36 $6^{\circ} 9^{\prime} \mathrm{N}$, 
$28^{\circ} 17^{\prime} \mathrm{E}, 65 \mathrm{~m}$, floodplain forest, Platanus litter, 5.VII.2002, leg. Assing (cAss); 1 ex., Muğla, $25 \mathrm{~km} \mathrm{NE}$ Fethiye, E Üzümlü, $36^{\circ} 44^{\prime} \mathrm{N}, 29^{\circ} 15^{\prime} \mathrm{E}, 610 \mathrm{~m}$, litter of Quercus ilex and shrubs, 4.X.2002, leg. Assing (cAss);

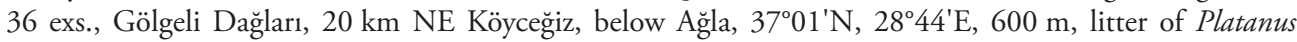
and other deciduous trees, 6.X.2002, leg. Assing (cAss). Antalya: 1 ex., Killik, cave entrance, 780 m, 2.-4. VI.2003, leg. Lohaj (cAss); 2 exs., N Kalkan, Dumanlı Dağı, 36 24'N, 2926'E, 1230 m, litter of Quercus ilex, 5.X.2002, leg. Assing (cAss); 1 ex., Termessos, 3.IV.1997, leg. Brachat (cSch); 1 ex., Alanya, Güzelbag, N Payallar, 24.III.1997, leg. Brachat (cSch); 1 ex., between Antalya and Alanya, Incekum, 16.IV.-2.V.1984, leg. Brachat (cSch); 1 ex., between Kumluca and Kemer, Tekirova, 2.IV.1997, leg. Brachat (cSch); 1 ex., NW Anamur, Ovabaşı near Köşekbaşı, 26.III.1997, leg. Brachat (cSch).

In the eastern Mediterranean, $H$. pisidicus is the most common representative of the genus. In Italy, it was previously known only from Puglia (Assing \& Wunderle 1995) and is here reported from Lazio, Basilicata, and Sardinia for the first time. The specimens from Corsica represent the first record from the island, as well as from France as a whole. There is little doubt that the record of $H$. ibericus from Corsica by Dauphin (2002) is erroneous and that it, too, refers to H. pisidicus, as can be inferred from the drawing of the male sternite VIII. Also, the species is here recorded from Croatia for the first time.

\section{Habrocerus ibericus Assing \& WUNDERLE}

Additional material examined:

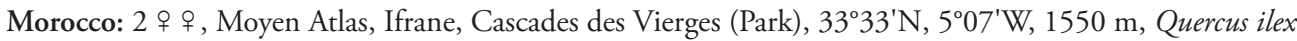
forest, 3.I.2002, leg. Bayer (cSch, cAss).

Spain: Andalucía: 1 ex., Cadiz, $14 \mathrm{~km} \mathrm{NE}$ Alcalá de los Gazules, 36 $32^{\prime} \mathrm{N} 5^{\circ} 39^{\prime} \mathrm{W}, 430 \mathrm{~m}$, oak forest, 2.II.1999, leg. Zerche (DEI); 2 exs., Cadiz, Canuto Garganta del Medio, 15 km NE Alcalá de los Gazules, $36^{\circ} 32^{\prime} \mathrm{N}, 5^{\circ} 38^{\prime} \mathrm{W}, 450 \mathrm{~m}$, litter of Rhododendron ponticum, 2.II.1999, leg. Zerche (DEI); 4 exs., Cadiz, ca. $10 \mathrm{~km} \mathrm{~W}$ Los Barrios, 36 $11^{\circ} \mathrm{N} 5^{\circ} 34^{\prime} \mathrm{W}, 210 \mathrm{~m}$, oak litter, 10.II.1999, leg. Zerche (DEI); 8 exs. [1 teneral], Cerro de la Novia, barranco at $\mathrm{km} 6$ of A-375, 36 $37^{\prime} \mathrm{N}, 5^{\circ} 30^{\prime} \mathrm{W}$, ca. $700 \mathrm{~m}$, collected together with H. capillaricornis, 19.II.2000, leg. Lompe (cAss); 1 ex., 15 km NNE Málaga, Montes de Málaga, $36^{\circ} 48^{\prime} \mathrm{N}, 4^{\circ} 22^{\prime} \mathrm{W}, 900 \mathrm{~m}$, mixed pine and corktree forest, 24.XII.2003, leg. Assing (cAss); 6 exs., ca. $25 \mathrm{~km}$ N Almuñécar, Sierra de Almijara, 36 $6^{\circ} 53^{\prime} \mathrm{N}, 3^{\circ} 42^{\prime} \mathrm{W}$, ca. $1200 \mathrm{~m}$, mixed pine, oak, and corktree forest, 25.XII.2003, leg. Assing (cAss); 1 ex., E Jaén, SE Mancha Real, Sierra Almadén, ca. 37²45'N, $3^{\circ} 33^{\prime} \mathrm{W}$, 1450-1850 m, Quercus ilex litter, 26.XII.2003, leg. Assing (cAss). Castilla y León: 2 exs., Montes Aquilianos S Ponferrada, 42 $26^{\prime} \mathrm{N}, 6^{\circ} 33^{\prime} \mathrm{W}, 1000 \mathrm{~m}, 7 . V I I I .2002$, leg. Behne (DEI, cAss); 1 ex., Sierra de la Demanda, ca. $40 \mathrm{~km}$ E Burgos, NE Valmala, Rábanos, $42^{\circ} 18^{\prime} \mathrm{N}, 03^{\circ} 16^{\prime} \mathrm{W}, 1190 \mathrm{~m}$, oak forest with grass, 12.X.2003,

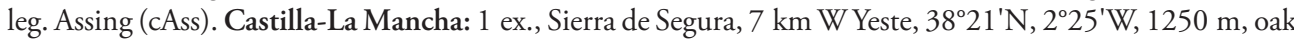
forest, 26.VII.2002, leg. Behne (DEI); 13 exs., Sierra de Alcaraz, ca. $15 \mathrm{~km}$ NNE Riópar, 38³2'N, $2^{\circ} 25^{\prime} \mathrm{W}$, 1350 m, stream bank, litter of Salix and other trees, 7.IV.2003, leg. Assing (cAss); 13 exs., Sierra de Alcaraz, ca. $15 \mathrm{~km}$ NNE Riópar, $38^{\circ} 35^{\prime} \mathrm{N}, 2^{\circ} 21^{\prime} \mathrm{W}, 1125 \mathrm{~m}$, litter of deciduous trees near stream, 10.IV.2003, leg. Assing (cAss); 2 exs., Sierra de Alcaraz, ca. 15 km NNE Riópar, $38^{\circ} 34^{\prime} \mathrm{N}, 2^{\circ} 20^{\prime} \mathrm{W}, 1120 \mathrm{~m}$, moss and litter near stream, 10.IV.2003, leg. Assing (cAss). Murcia: 1 ex., Sierra de Espuña, 1500 m, 7.VI.2003, leg. Forcke (cAss). Aragón: 70 exs., $50 \mathrm{~km}$ ESE Teruel, $10 \mathrm{~km} \mathrm{~N}$ Rubielos de Mora, 40¹4'N, 0³8'W, $1255 \mathrm{~m}$, oak forest, 13.IV.2003, leg. Assing (cAss); 1 ex., WNW Teruel, Sierra de Albarracín, NE Noguera, 40³0'N, $1^{\circ} 35^{\prime} \mathrm{W}, 1625$ m, NE-slope, oak forest, 11.IV.2003, leg. Assing (cAss). Islas Baleares: 1 ex., Mallorca, Lluc Coma Freda, 800 m, 24.III.2003, leg. Feldmann (cAss).

Portugal: 8 exs., Algarve, Serra de Monchique, Portela Viuva, 20.-21.II.1999, leg. Meybohm (cAss); 1 ex., Serra de Monchique, N Foia, 850 m, 18.II.1999, leg. Meybohm (cAss).

The distribution of $H$. ibericus is confined to the Western Mediterranean. The species is not rare in the Iberian peninsula, but apparently very local in southern France. As mentioned in the preceding section, the record of H. ibericus from Corsica by DAupHIN (2002) probably refers to 
H. pisidicus. The specimens from Morocco would represent the first record from North Africa, but, since they are females, the identification is somewhat uncertain. Male-based records would be required to confirm the presence of $H$. ibericus in North Africa.

\section{Habrocerus canariensis AsSING \& WUNDERLE}

Additional material examined:

Canary Islands: Gran Canaria: 1 ex., Bco. Cernicalos, 4.II.1989, leg. García (cGar). El Hierro: 5 exs. [1 teneral], Lomo Blanco, 6 km W Frontera, 550 m, fayal-brezal, 25.I.1998, leg. Behne (DEI, cAss); 2 exs., Las Puntas, $27^{\circ} 48^{\prime} \mathrm{N}, 17^{\circ} 59^{\prime} \mathrm{W}, 50 \mathrm{~m}$, fallow, in rotting plant debris, 4.I.2000, leg. Assing (cAss); 3 exs., El Golfo, laurisilva, leg. Franz (NHMW); 4 exs., Mocanal near Valverde, leg. Franz (NHMW). La Gomera: 3 exs. [2 teneral], N Roque de Agando, El Bailadero, 1050 m, laurisilva with Erica, 24.XII.1998, leg. Assing (cAss); 20 exs., El Cedro, E Ermitá N. S. de Lourdes, 1000 m, laurisilva, 24.XII.1998, leg. Assing (cAss); 29 exs., El Cedro, near Ermitá N. S. de Lourdes, 900-1000 m, laurisilva, 24.\&27.XII.1998, leg. Assing (cAss); 4 exs., NE Arure, Araña, 1000-1100 m, moist litter in laurisilva, 24.XII.1998, leg. Assing (cAss); 1 ex., N La Laguna Grande, barranco with laurisilva, 1250 m, 28.XII.1998, leg. Assing (cAss). La Palma: 3 exs., SW Franceses, Bco. de Los Hombres, $800 \mathrm{~m}$, litter of Castanea and Erica, 1.IV.1999, leg. Assing (cAss); 1 ex., Roque Faro, 1000 m, Pinus wood with Myrica undergrowth, 1.IV.1999, leg. Assing (cAss); 1 ex., Barlovento, leg. Franz (NHMW).

In contrast to La Gomera, the species appears to be very rare in La Palma and it seems to be absent from mature laurisilva, where only $H$. capillaricornis was observed. In El Hierro, H. canariensis was collected in rotting debris (together with several staphylinid species typically occurring in compost) at an altitude of only $50 \mathrm{~m}$ above sealevel; it was not found in the laurel woods at altitudes above $600 \mathrm{~m}$, where it usually occurs in La Gomera and La Palma.

\section{Habrocerus cyprensis Assing \& WUNDERLE}

Additional material examined:

Turkey: 1 ơ 1 \% , Antalya, Alanya, Kuzyaka, bank of Dim Çayóriver, 10 m, sifted from debris, 12.III.2000, leg. Rose (cRos, cAss).

The species was previously known only from Cyprus and Rhodos (Assing \& Wunderle 1995, 1996). The above specimens represent the first record from Turkey.

\section{Habrocerus simulans Assing \& WUNDERLE}

Additional material examined:

Turkey: Ordu: 3 exs., N-Anatolia, Ünye, 17.V.1987, leg. Rozner (cAss). Mersin: 26 exs., Çamlıyayla, $37^{\circ} 10^{\prime} \mathrm{N}$, $34^{\circ} 35^{\prime} \mathrm{E}, 1150 \mathrm{~m}, 3 . \mathrm{V} .2004$, leg. Besuchet (cAss). Adana: 1 ex., Adana, Karatepe, 37 ${ }^{\circ} 17^{\prime} \mathrm{N}, 36^{\circ} 14^{\prime} \mathrm{E}, 200 \mathrm{~m}$, laurisilva, 24.IV.-1.V.2002, leg. Meybohm \& Brachat (cAss). Osmaniye: 1 ex., SE Osmaniye, Zorkum, $36^{\circ} 58^{\prime} \mathrm{N}, 36^{\circ} 22^{\prime} \mathrm{E}, 1670 \mathrm{~m}, 29 . \mathrm{IV} .2004$, leg. Meybohm \& Brachat (cAss); 1 ex., $13 \mathrm{~km}$ E Osmaniye, NW Yarpuz, $37^{\circ} 08^{\prime} \mathrm{N}, 36^{\circ} 25^{\prime} \mathrm{E}, 930 \mathrm{~m}$, beech and Platanus forest, 6.IV.2004, leg. Assing (cAss); 5 exs., E

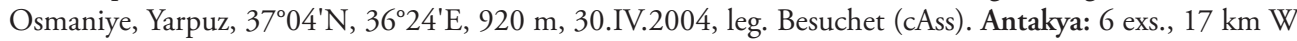
Antakya, NW Teknepinar, $36^{\circ} 11^{\prime} \mathrm{N}, 35^{\circ} 59^{\prime} \mathrm{E}, 400 \mathrm{~m}$, pine forest with oak and shrubs, 3.IV.2004, leg. Assing (cAss); 1 ex., Harbiye, $9 \mathrm{~km} \mathrm{SSW}$ Antakya, $36^{\circ} 08^{\prime} \mathrm{N}, 36^{\circ} 08^{\prime} \mathrm{E}, 430 \mathrm{~m}$, bank of stream, laurel and Platanus litter, 5.IV.2004, leg. Assing (cAss); 1 ex., Iskenderun, Belen, 36 $30^{\prime} \mathrm{N}, 36^{\circ} 11^{\prime} \mathrm{E}, 340 \mathrm{~m}$, 29.IV.2002, leg. Meybohm \& Brachat (cAss); 5 exs., Ziyaret Dağ, W Sungur, 360' N, 36 05'E, 760 m, 21.IV.2004, leg. Besuchet, Brachat \& Meybohm (cAss). 
Lebanon: 1 ex., Fnaideq, Qammouaa forest, $34^{\circ} 29^{\prime} \mathrm{N}, 36^{\circ}{ }^{\circ} 15^{\prime}$, 1300-1600 m, 26.V.2006, leg. Frenzel (cAss).

The species was previously recorded from Israel, Lebanon, and eastern Anatolia (Assing \& Wunderle 1995). The records from Bolu and Mersin considerably extend the known range of the species further to the west and northwest.

\section{Habrocerus indicus AssING \& WUNDERLE}

Additional material examined:

Nepal: 2 exs., Annapurna South Himal, SE Narcheng, left river side of Bele Khola, $28^{\circ} 31^{\prime} \mathrm{N}, 83^{\circ} 42^{\prime} \mathrm{E}$, 2300 m, 25.-26.V.2001, leg. Hirthe (cHir, cAss).

The known distribution of Habrocerus indicus is confined to northern India and Nepal (Assing \& Wunderle 1995, 1996).

\section{Habrocerus splendens sp. $\mathbf{n}$.}

(Figs 1-11, Map 1)

\section{Type material:}

Holotype $0^{\star}$ : "China: Yunnan [CH07-17], Baoshan Pref., mountain range $25 \mathrm{~km}$ S Tengchong, $1900 \mathrm{~m}, 24^{\circ} 48^{\prime} 28^{\prime \prime N}, 98^{\circ} 32^{\prime} 03^{\prime \prime E}$, dev. primery [sic] decid. forest, litter, fungi sifted, 2.VI.2007, M. Schülke / Holotypus ơ Habrocerus splendens sp. n. det. V. Assing 2007" (cAss). Paratypes: 1 : : "China: Yunnan [CH07-18], Baoshan Pref., mountain range $22 \mathrm{~km} \mathrm{~S}$ Tengchong, $1750 \mathrm{~m}$, $24^{\circ} 49^{\prime} 29^{\prime \prime N}, 98^{\circ} 29^{\prime} 27^{\prime \prime E}$, second. forest, litter, dead wood sifted, 2.VI.2007, M. Schülke" (cSch); 1 ơ, 1 9 : "China: Yunnan [CH07-18], Baoshan Pref., mountain range $22 \mathrm{~km} \mathrm{~S}$ Tengchong, $1750 \mathrm{~m}, 24^{\circ} 49^{\prime} 29^{\prime \prime} \mathrm{N}, 98^{\circ} 29^{\prime} 27^{\prime \prime} \mathrm{E}$, second. forest, litter, dead wood sifted, 2.VI.2007, leg. A. Pütz" (cPüt, cAss).

\section{Description:}

3.4-3.7 mm. External morphology as in $H$. capillaricornis, except as follows:

Antennae with antennomeres I-II pale brown to dark brown and III-XI pale yellowish. Pronotum conspicuously glossy, without trace of microsculpture even near the margins (Fig. 1). Abdomen with relatively sparse fine puncturation and with very shallow, barely noticeable microsculpture (Fig. 2).

$0^{*}$ : tergite and sternite VII large, with broadly and weakly convex posterior margins (Figs 3-4); sternite VIII posteriorly with deep and proximally U-shaped excision; appendices of pleurites VIII each with one long seta (Fig. 5); sclerites of segment IX as in Fig. 6; internal sac of aedeagus with longer row of numerous strongly sclerotised structures and with additional row of 8 slender spines (Fig. 7).

ㅇ: tergite and sternite VII distinctly smaller than in $\sigma^{\star}$; posterior margin of tergite VII acute, that of sternite VII strongly convex (Figs 8-9); segment VIII as in Fig. 10.

\section{Comparative notes:}

Based on the morphology of the primary and secondary sexual characters, the species refers to the H. capillaricornis species group, whose original distribution is confined to the Palaearctic region. From all other species of this group, $H$. splendens is distinguished by the sparser puncturation and 
the less distinct microsculpture of the abdomen, as well as by the distinctive internal structures of the internal sac and by the secondary sexual characters (shapes of male sternite VIII, as well as of female tergite and sternite VII). For illustrations of the sexual characters of other species of the H. capillaricornis group see AssIng \& WUNDERLE (1995). The only other Habrocerus species that was previously known from China is H. schuelkei Assing \& Wunderle, 1996 from Shaanxi. This species, however, refers to the $H$. schwarzi species group and is characterised by sexual characters of completely different morphology; for illustrations see Assing \& WUNDERLE (1996).

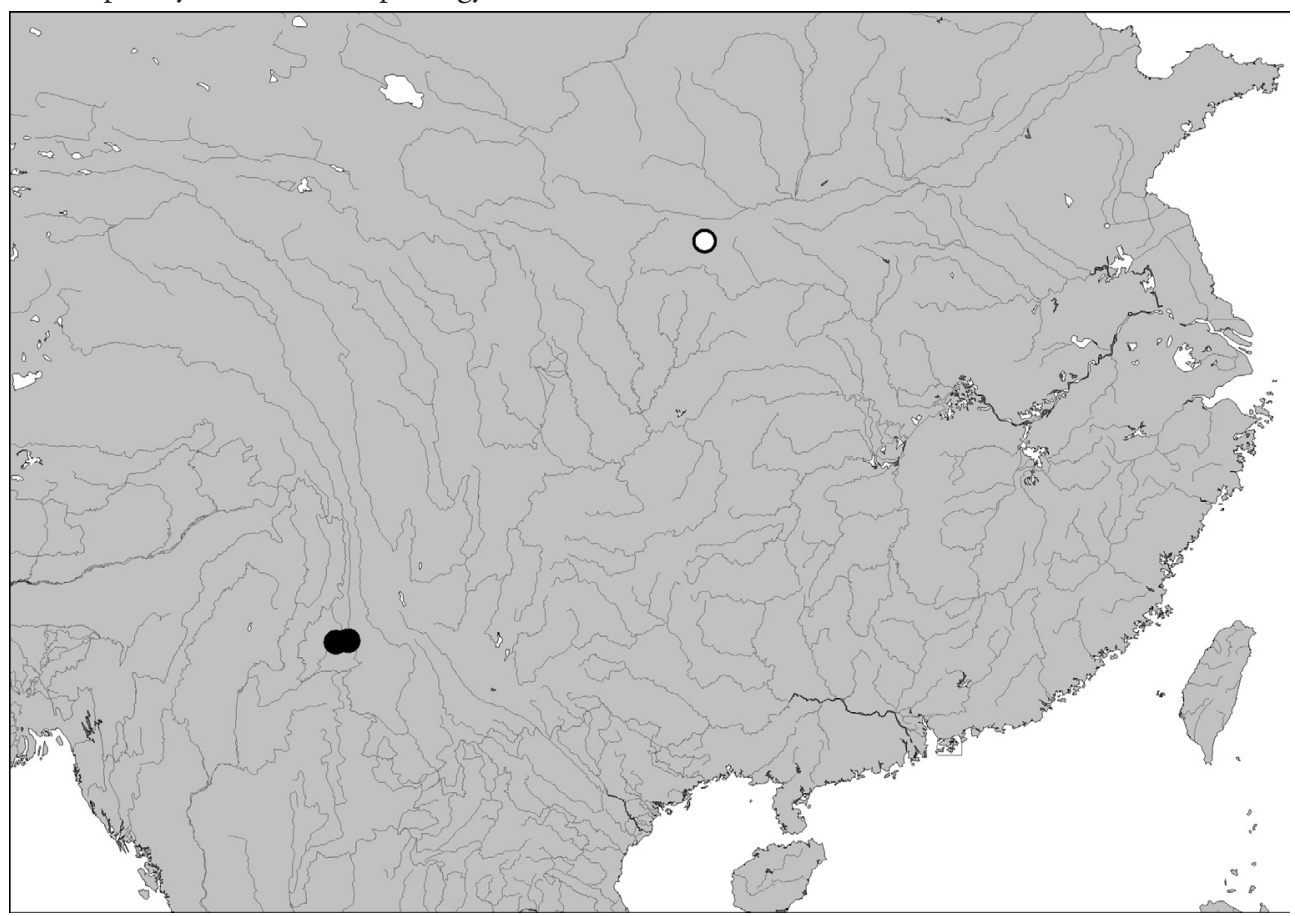

Map 1: Known distribution of the genus Habrocerus in China: Habrocerus splendens sp. n. (filled circles) and H. schuelkei Assing \& Wunderle (open circle).

\section{Etymology:}

The name (Latin, present participle of splendere) refers to the glossy appearance of the species.

\section{Distribution and bionomics:}

The type specimens were collected in two localities to the south of Tengchong (China: Yunnan province) by sifting the leaf litter of deciduous forests at altitudes of 1750 and $1900 \mathrm{~m}$. The abdomen of a dissected female paratype contained a conspicuous structure of unknown identity (Fig. 11).

\section{Habrocerus costaricensis AsSING \& WUNDERLE}

Additional material examined:

Costa Rica: 1 ơ $^{\star}$ San Antonio de Escaza, 1300 m, XI.-XII.1997, leg. Eberhard (cSch).

The species has become known only from Costa Rica (Assing \& Wunderle 1995); the above specimen represents the first record since the original description. 

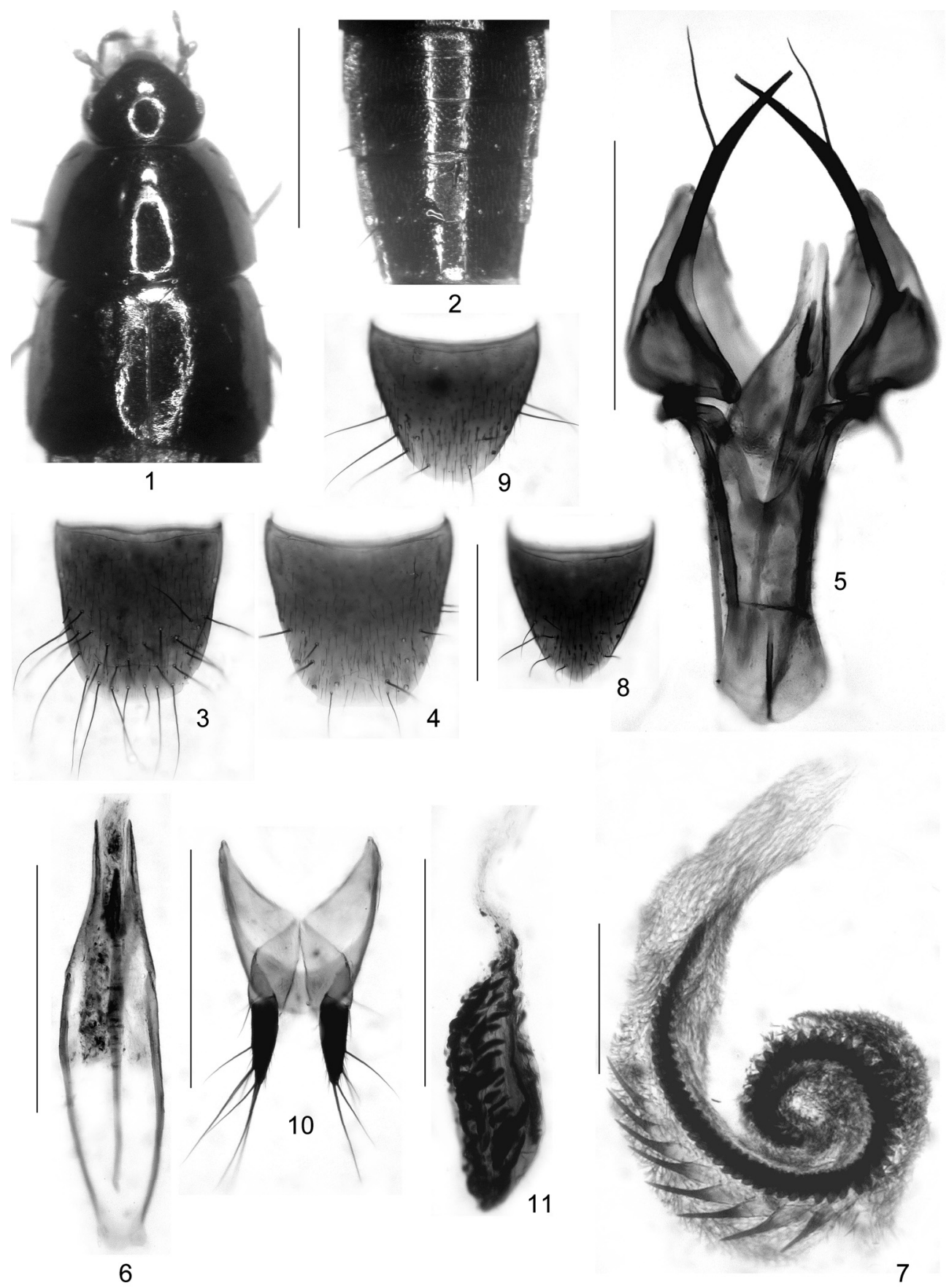

Figs 1-11: Habrocerus splendens sp. n.: forebody (1); abdominal segments III-VI (2); male tergite VII (3); male sternite VII (4); male segments VIII-IX (5); sclerites of male segment IX (6); internal sac of aedeagus (7); female tergite VII (8); female sternite VII (9); female segment VIII (10); unidentified structure in female abdomen (11). Scale bars: 1-2: $1.0 \mathrm{~mm}$; 3-6, 8-11: $0.5 \mathrm{~mm}$; $7: 0.2 \mathrm{~mm}$. 


\section{Nomimocerus longispinosus Assing \& WUNDERLE}

Additional material examined:

Chile: 15 exs., Aisén prov., Parque Nat. Rio Simpson, leg. Franz (NHMW, cAss).

The species is known from Aisén and Chiloé provinces, Chile (Assing 1998, Assing \& Wunderle 1995).

\section{Nomimocerus peckorum AsSING \& WUNDERLE}

Additional material examined:

Chile: 6 exs., Osorno, Parque Nacional Puyehue, leg. Franz (NHMW, cAss); 3 exs., Aisén prov., Coihaique, leg. Franz (NHMW, cAss).

The record from Coihaique extends the known range of the species further to the south by some $500 \mathrm{~km}$. Previously, N. peckorum was known from Osorno (including Valdivia), Llanquihue, and Cautin provinces (Assing 1998, Assing \& Wunderle 1995).

\section{Acknowledgements}

My thanks are due to the colleagues indicated in the material section for arranging a loan of material under their care. In particular, I am grateful to Michael Schülke for the generous gift of the holotype of H. splendens. Benedikt Feldmann proof-read the manuscript.

\section{References}

Assing, V. 1998: A revision of the Habrocerinae of the world. Supplement II (Coleoptera: Staphylinidae). - Revue Suisse de Zoologie 105: 487-492.

Assing, V. \& Wunderle, P. 1995: A revision of the species of the subfamily Habrocerinae (Coleoptera: Staphylinidae) of the world. - Revue Suisse de Zoologie 102: 307-359.

Assing, V. \& Wunderle, P. 1996: A revision of the species of the subfamily Habrocerinae of the world. Supplement I. (Coleoptera: Staphylinidae). - Beiträge zur Entomologie, Berlin 46: 373-378.

Assing, V. \& Wunderle, P. 1999: On the Staphylinidae of La Palma, Canary Islands, with descriptions of new species and remarks on some Canarian Stenus Latreille (Coleoptera). - Entomologische Zeitschrift 109: 433-443.

Dauphin, P. 2002: Sur les localités françaises de Habrocerus ibericus Assing \& Wunderlé [sic] (Coleoptera Staphylinidae Habrocerinae). - Bulletin de la Société linnéenne de Bordeaux 30: 153-155.

Franz, H. 1996: Die Ergebnisse meiner langjährigen Aufsammlungen der Coleopteenfauna [sic] auf der Insel Hierro (Kanarische Inseln). - Sitzungsberichte der Österreichischen Akademie der Wissenschaften, Mathematisch-Naturwissenschaftliche Klasse, Abteilung I 202 (1995): 71-138.

Herman, L. H. 2001: Catalog of the Staphylinidae (Insecta: Coleoptera). 1758 to the end of the second millennium. Volumes I-VII. - Bulletin of the American Museum of Natural History 265: 4218 pp.

Newton, A. F. 2006: Habrocerus capillaricornis (Gravenhorst) (Coleoptera: Staphylinidae) discovered in South Africa. - African Entomology 14: 193-194.

Author's address:

Dr. VOLKer Assing

Gabelsbergerstr. 2

30163 Hannover, Germany

e-mail: vassing.hann@t-online.de
Subject editor:

Dr. L. ZerChe 